\title{
Rats’ Metabolic Responses to Chronic Intervention with Cantonese Herbal Tea
}

Rong You ${ }^{12^{*}}$, Liang Yin ${ }^{1}$, Yanqing Guan ${ }^{1}$ and Lin $\mathrm{Li}^{2^{*}}$

${ }^{1}$ College of Life Sciences, South China Normal University, Guangzhou, PR China

${ }^{2}$ College of Light Industry and Food Sciences, South China University of Technology, Guangzhou, PR China

\begin{abstract}
Cantonese Herbal Tea (CHT) is consumed in south China to alleviate feelings of discomfort due to the over-heat and humidity in the body, but the related molecular mechanisms behind its effects remain obscure. The urinary metabolic responses to $\mathrm{CHT}$ over time in rats were investigated during a 38-day-period using GC-MS-based metabonomics. Integrated with student's t-test and Orthogonal Projection on Latent Structures Discriminant Analysis (OPLS-DA) at each time point revealed that the process could be divided into two stages. In the first stage, the bioefficacy was characterized by elevated antioxidant activity and the depletion of oxidative stresses. However, the effect was reduced with time and replaced by a second stage characterized by the decreased flow of energy metabolism and an imbalance in gut microbiota. In conclusion, metabonomics study gained insight into the metabolic changes induced by $\mathrm{CHT}$ intake and the extent to which $\mathrm{CHT}$ intake influences the urinary metabolome, which also provides a powerful method for understanding the mechanism of $\mathrm{CHT}$.
\end{abstract}

Keywords: CHT; GC-MS; Metabonomics; Metabolic responses

\section{Introduction}

Cantonese Herbal Tea (CHT) is an aqueous extract of a mixture of medicinal and edible plants and has a long history of consumption in South China. According to Traditional Chinese Medicine (TCM) theory, dietary intervention with $\mathrm{CHT}$ can detoxify and drive sensations of heat and humidity from the body, which is considered to be an adaptation to local climatic conditions with subtropical weather characterized by excessive heat and humidity [1]. It has been refined as a functional and complementary drink to alleviate the symptoms of stresses [2]. However, the high complexity in the composition of herbal tea, the multi-level biological effects as well as the subtle magnitude of modulating effects on, have posed obstacles for investigating and evaluating the molecular mechanisms behind the medicinal properties of herbal tea [2-6]. Therefore, its beneficial and adverse effects are substantially more difficult to monitor with reasonable precision using a reductionist approach [7].

As a holistic and systematic biological approach, metabonomics involves the comprehensive study of molecular metabolites in a biological system under specified conditions and the interpretation of changes on metabolic pathways to decipher the physiological variation in the whole organism [8]. Metabonomics has been found widespread applications in many areas including diseases, drugs, nutrition and plant etc. [9-12]. In comparison of genomics and proteomics, metabonomics enables the monitoring of real end-point metabolic changes following physiological and pathophysiological stimuli [13] and interpret holistic, quantitative data on time-dependent metabolic variation in response to a xenobiotic intervention, without prior knowledge of its composition. Recently, considerable efforts are being expended to enhance the understanding of the mechanisms of and individual responses to dietary intervention with the aid of metabonomics [14-16]. However, current studies are still relatively simple because the active ingredients have known biochemical targets. Studies of CHT may be even further complicated by the facts that its complex constituents have unknown biochemical targets and the overall metabolic response to $\mathrm{CHT}$ will be the result of complex interactions.

Metabonomics involves the large-scale analysis of metabolites using high-throughput techniques and bioinformatics tools for data analysis, visualization, and integration. High-throughput analysis should be global, quantitative, robust, reproducible, accurate and interpretable. Hyphenated Gas Chromatography-Mass Spectrometry (GC-MS) is an analytical method that in recent years has evolved to enable simultaneous analysis of many different metabolites in urine samples due to its high resolution and high sensitivity $[17,18]$. Additionally, biological processes (such as metabolic response) are dynamic by nature and have temporal progressions and dynamic sampling will improve the detection of changes that occur over time following treatment. Thus, in this study a GC-MS-based metabonomics investigation has been performed to track subtle, time-dependent fluctuations of metabolites that occurred in response to $\mathrm{CHT}$ and to unravel the complex relationship between the consumption of $\mathrm{CHT}$ and its effects on health in rats over a 38-day chronic intervention period with an expectation that this treatment would produce cumulative effects $[19,20]$.

\section{Materials and Methods}

\section{Animal treatment}

The animal experiment was performed at the SPF (Specific Pathogen Free) animal laboratory at the Shenzhen Research Institute of Hong Kong University of Science \& Technology, which has been certified by the Guangdong Science and Technology Committee with a laboratory animal facility certificate in accordance with the National Standard for the Quality of Laboratory Animals in China. Nineteen healthy male SPF (specific pathogen-free) Sprague Dawley (SD) rats approximately

*Corresponding authors: Rong You, College of Life Sciences, South China Normal University, Guangzhou, PR China, Tel: 86-20-85211372; E-mail: yourong@scnu.edu

Lin li, College of Light Industry and Food Sciences, South China University of Technology, Guangzhou, PR China, Tel: 86-20-87110249; E-mail: felirnli@scut.edu.cn

Received May 28, 2016; Accepted June 15, 2016; Published June 20, 2016

Citation: You R, Yin L, Guan Y, Li L (2016) Rats' Metabolic Responses to Chronic Intervention with Cantonese Herbal Tea. Metabolomics 6: 178. doi:10.4172/2153 0769.1000178

Copyright: (c) 2016 You R, et al. This is an open-access article distributed under the terms of the Creative Commons Attribution License, which permits unrestricted use, distribution, and reproduction in any medium, provided the original author and source are credited. 
3 months of age and weighing $200 \pm 20 \mathrm{~g}$ were purchased from the Guangdong Experimental Animal Center. All animals were housed in individual cages and had free access to water and food throughout the experiment. The light cycle consisted of $12 \mathrm{~h}$ of light and $12 \mathrm{~h}$ of dark, and the temperature was maintained at $22-24^{\circ} \mathrm{C}$. After 7 days of acclimatization, animals were transferred to individual metabolic cages (one animal per cage) for urine collection. The rats were randomly divided into a control group $(n=9)$ and a treated group $(n=10)$. The treated group was orally fed $0.4 \mathrm{mg} \cdot \mathrm{g}^{-1}$ of CHT daily for 38 days, while control rats received the same amount of water. Urine samples were collected at days $0,8,14,20,26,33$ and 38 during the Herbal Tea treatment period. Precipitated solids were removed by centrifugation at $12,000 \mathrm{rpm}$, and urine samples were stored at $-80^{\circ} \mathrm{C}$ prior to GC-MS analysis.

\section{Sample preparation}

Urine samples were thawed and $100 \mu$ of urine was incubated with $600 \mathrm{U}$ urease (Type C-3, Sigma) at $37^{\circ} \mathrm{C}$ for $1 \mathrm{~h}$. Then, $300 \mu \mathrm{l}$ of ice-cold methanol was added and mixed vigorously. The mixture was placed on ice for $20 \mathrm{~min}$, followed by centrifugation to precipitate urease and protein. A total of $200 \mu$ of the supernatant was separated and evaporated until dry under a gentle stream of nitrogen gas. A total of $100 \mu$ l of toluene (dried over anhydrous sodium sulfate) was added to the dry residue and then mixed and dried again under nitrogen gas. The dried extract was derivatized first with $30 \mu \mathrm{l}$ of dry pyridine $(20 \mathrm{mg} / \mathrm{ml})$ for $16 \mathrm{~h}$ at $37^{\circ} \mathrm{C}$, followed by trimethylsilyl-derivatization with $120 \mu \mathrm{l}$ of BSTFA with $1 \%$ TMCS (Sigma) at $70^{\circ} \mathrm{C}$ for $2 \mathrm{~h}$. A volume of $50 \mu \mathrm{l}$ of heptane was added after silylation.

\section{GC-MS analysis of urine}

Urine samples were analyzed by GC-MS (Agilent 6890 GC instrument equipped with a 5975 mass detector), using a HP-5 ms GC column ( $30 \mathrm{~m}$ length, $0.25 \mathrm{~mm}$ i.d. and $0.20 \mu \mathrm{m}$ film thickness). The sample injection ( $1 \mu \mathrm{l}$ volume) was split less at an injection temperature of $250^{\circ} \mathrm{C}$. Helium carrier gas flow was $1.0 \mathrm{ml} / \mathrm{min}$. The ion source temperature was $230^{\circ} \mathrm{C}$. The temperature gradient of GC separation started at $70^{\circ} \mathrm{C}$ for $2 \mathrm{~min}$, increased from $70^{\circ} \mathrm{C}$ to $140^{\circ} \mathrm{C}$ at $7^{\circ} \mathrm{C} / \mathrm{min}$ where it remained for $2 \mathrm{~min}$, then increased again from $140^{\circ} \mathrm{C}$ to 210 at $4^{\circ} \mathrm{C} / \mathrm{min}$. The $210^{\circ} \mathrm{C}$ temperature was held for $4 \mathrm{~min}$, then increased from $210^{\circ} \mathrm{C}$ to $280^{\circ} \mathrm{C}$ at $7{ }^{\circ} \mathrm{C} / \mathrm{min}$ where it remained for $7 \mathrm{~min}$. Metabolites were identified by matching mass spectra to the NIST05 library. The HMDB metabolomics database was also used for compound identification and KEGG was used for pathway analysis.

\section{Data preprocessing}

Raw GC-MS files were converted into NetCDF format using Chemeostation software (Agilent Inc., USA). The XCMS package contains three important algorithms for peak detection, peak matching, and retention time alignment and was used to deconvolute and align the NetCDF files into a single data set [21]. XCMS parameters were set as follows: a full-width half-maximum of 4 , a snthresh of 6 , a bandwidth of 8 for the first grouping command and 4 for the second grouping command.

A three-dimensional matrix including peak index $(\mathrm{m} / \mathrm{z}$-retention time, $m / z-\mathrm{RT}$ ), sample names and peak areas was exported from XCMS in "tsv" format, which can be viewed using Microsoft Excel. Artifacts arising from the BSTFA derivatizing reagent were removed. The $\mathrm{m} / \mathrm{z}$ ions of 73,75 , and 147 were also excluded because many TMS derivatives produce these typical fragments. As variations in overall urinary concentrations were very distinctive, normalization to the total sum of the ion fragments was performed prior to pattern recognition analysis.

\section{Multivariate statistical data analysis}

Normalized data were introduced into SIMCA-P 11.5 Software for multivariate statistical analysis. Mean centering calculates the average spectrum of the data set and subtracts the average from each spectrum, with the goal of focusing on the fluctuating part of the data instead of the original value. Pareto scaling weighs each variable by the square root of its standard deviation, which amplifies the contribution of lower concentration metabolites but not to such an extent that noise makes a large contribution [22]. Thus, Principal Component Analysis (PCA) was initially performed on mean-centered data to visualize general clustering and trends among the observations. Orthogonal projection on latent structures discriminant analysis (OPLS-DA) was performed on Pareto scaled data to maximize the separation and identify biomarkers that differed between samples at different sampling time points, where the normalized peak data were defined as $X$ variables with interventions, i.e., not-fed or fed CHT, as $Y$ variables.

\section{Model validation and biomarker selection in OPLS-DA models}

A 7-fold cross validation strategy was applied to check the model validity and produce the cross validation parameter $\mathrm{Q}^{2}$. Permutation testing was also conducted to validate the predictability of the model [23]. If the maximum $\mathrm{Q}^{2}$ obtained from the permutated models comparing controls and treatment-related groups did not exceed $\mathrm{Q}^{2}$ obtained from the real model, the model suggested great predictability. The metabolites associated with CHT intervention could be identified and considered statistically significant according to the Variable Importance Parameter (VIP) obtained from the OPLS-DA models using a combination of Student's $t$-tests $(\mathrm{p}<0.05)$. A positive or negative change is indicated by the change in the direction of the identified metabolites relative to zero in the OPLS-DA coefficient plot; positive plots indicate an increased concentration and negative plots indicate a decreased concentration, compared to controls.

\section{Results}

\section{GC-MS profiling of urinary metabolite profiles}

A representative GC-MS total ion chromatogram of a urine sample is shown in SI Figure 1. There was a large dynamic range in metabolite concentration. Metabolites were identified by mass spectra matches to the GC-MS mass spectral library NIST05 and the HMDB metabolomics database GC/MS library, which contains 318 small molecule metabolites found in the human body. In all, approximately 67 compounds were confirmed, including carboxylic acids, hydroxyl acids, carbohydrate, phenolics, amino acids and amines (SI Table 1). Because there was variation in the concentration between urine samples as well as interand intra-individual physiological variation, visual examination of the GC-MS chromatograms from the urine samples did not reveal obvious distinguishing features between dosed rats and controls.

\section{Data analysis}

Normalization to the total sum of the ion fragments was performed before multivariate analysis to compensate for the variation in overall concentrations between urine samples. PCA was initially performed to detect overall trends within the multivariate data, using all of the normalized urine data, which consists of 149 observations and 175 variables. Two components were calculated for the model, where the 


\begin{tabular}{|c|c|c|c|c|}
\hline $\begin{array}{c}\text { Sampling } \\
\text { points } \\
8 \mathrm{~d}\end{array}$ & \multicolumn{3}{|c|}{ OPLS-DA } & $\begin{array}{c}\text { Permutation test } \\
\mathrm{Q} 2 \mathrm{M} A \mathrm{Q}<\mathrm{Q} 2\end{array}$ \\
\cline { 2 - 5 } & $\mathrm{R}^{2} \mathrm{X}$ & $\mathrm{R}^{2} \mathrm{Y}$ & $\mathrm{Q}^{2}$ & $\mathrm{Q}^{2} \mathrm{MAX}<\mathrm{Q}^{2}$ \\
\hline day 8 & 0.355 & 0.907 & 0.579 & $\mathrm{Q}^{2} \mathrm{MAX}<\mathrm{Q}^{2}$ \\
\hline day 14 & 0.376 & 0.932 & 0.785 & $\mathrm{Q}^{2} \mathrm{MAX}<\mathrm{Q}^{2}$ \\
\hline day 20 & 0.426 & 0.967 & 0.813 & - \\
\hline day 26 & 0.325 & 0.813 & 0.371 & $\mathrm{Q}^{2} \mathrm{MAX}>\mathrm{Q}^{2}$ \\
\hline day 33 & 0.41 & 0.596 & -0.21 & - \\
\hline day 38 & 0.364 & 0.865 & 0.523 & $\mathrm{Q}^{2} \mathrm{MAX}<\mathrm{Q}^{2}$ \\
\hline
\end{tabular}

$R^{2} X$ and $R^{2} Y$ are the variation in $X$ and $Y$ matrix, respectively, and $Q^{2}$ indicates the predictability of the model. Values of these parameters close to 1.0 indicate a robust and reliable model. $\mathrm{Q}^{2} \mathrm{MAX}<\mathrm{Q}^{2}$ means the model is valid and predictable based on a permutation test and vice versa

Table 1: Parameters for the assessment of modeling quality.

time-dependent metabolic alterations of control rats and dosed rats were globally observed. The trajectories of the metabolic profiles at different time points showed that both groups moved along the direction of the first group. However, inter-individual and intra-individual variation dominated the PCA analysis to such an extent that it was not possible to separate the CHT group from the controls.

Hence, OPLS-DA was performed to identify the metabolites that could separate the control group from the group treated with $\mathrm{CHT}$ at different time points using Pareto scaling and the scatter plots were shown in Figure 1. This supervised technique was applied to sharpen the separation between groups of observations, by rotating PCA components such that a maximum separation among classes is obtained, and to understand which variables carry the class-separating information and therefore define biomarkers. The $\mathrm{R}^{2}$ and $\mathrm{Q}^{2}$ values were employed as first indicators of the OPLS-DA model quality and are listed in Table 1. Strong separations were achieved along the first component direction when comparing controls and treatments at days 8, 14, 20, 26 and 38, respectively, but the OPLS-DA model for comparisons between the $\mathrm{CHT}$ and control groups at days 33 produced a negative $\mathrm{Q}^{2}$, which indicated poor predictability and was thus discarded. To further validate OPLS-DA models, permutation tests were performed. A higher $\mathrm{Q}^{2} \max$ was obtained from the permutation test than from the real model in the comparison of the control group versus the CHT group at 26 days postdose, implying that the model lacked predictability and was invalid. All of the other models produced had good predictability, as suggested in Table 1. Those only weak models could be obtained between the control and treated rats at days 26 and 33 suggested that treated animals regained homeostatic balance or that their metabolic fluctuations following CHT intervention remained within the normal range.

\section{Biomarkers selection}

To transform the set into biochemical information, interpretation was necessary, which included the mining of complex data to identify metabolites that were statistically significant and thus potentially biochemically interesting compounds. Metabolites responsible for the separation of treated rats from controls at days 8, 14, 20 and 38 postdose could be extracted according to their relative influence on the model using the variable importance parameter. Variables with VIP $>1$ were considered significant in this model.

The OPLS-DA loadings revealed 26 up and down regulated metabolites that were responsible for differentiation between the $\mathrm{CHT}$ and control groups at days 8 post-dose. These metabolites may be involved in energy metabolism (succinic acid, citric acid and lactate), amino acid metabolism (alanine, proline, $\mathrm{N}$-acetylglutamate,
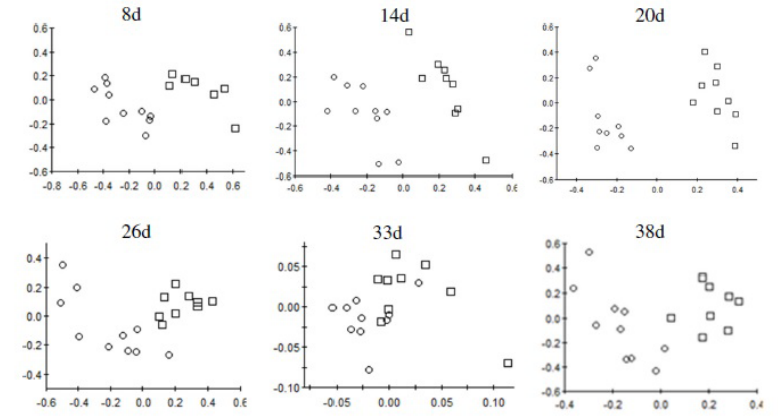

Figure 1: OPLS-DA score plots between herbal tea group and contro group at different sampling points.

methylglycine), taurine and hypotaurine metabolism (hypotaurine), lysine degradation, and other metabolisms. The same procedures were applied to the GC-MS chromatographs at days 14, 20 and 38 pose-dose. The perturbed metabolites, their variable importance parameters and directional changes and the metabolic pathways involved are listed in Table 2. The OPLS-DA loadings of the models at all sampling time points indicated that lactate, alanine, and benzoic acid were responsible for differentiation at all sampling time points and other metabolites changed at some time points but returned to normal at other time points. The levels of lactate and benzoic acid were reduced at the first two sampling time points, increased on day 20 and decreased again on day 38, compared to controls. An initial elevation in alanine was observed on day 8 , and decreased at later sampling time points.

Furthermore, student's t-test combined with VIP statistics was used for choosing the most significant and reliable variables to avoid overestimation of themetabolites that werepresentinhigh concentrations and their correspondingly high variances [24]. Integrating the OPLSDA loadings with student's $t$-test for the model comparing the urinary spectra of the CHT group rats and the controls at days 8 detected several significantly perturbed metabolites, including elevated levels of alanine, $\mathrm{N}$-acetylglutamate, lysine and hypotaurine and decreases in the level of glycolic acid. Urine samples obtained from the rats at days 20 after CHT intake were typified by increased concentrations of erythritol. On day 38 , increasingly significant differentiation between the intervention group and the controls was obtained again, as suggested by a positive $\mathrm{Q}^{2}$, with elevated levels of indole-3-acetic acid, galactose and palmitic acid and depleted levels of hypotaurine, glyceric acid. All metabolites responsible for differentiation at days $8,14,20$ and 38 are listed together in Table 2.

\section{Discussion}

In the present study, metabonomics was utilized to investigate the metabolic response to $\mathrm{CHT}$ intervention in rats. It is commonly believed that the effects of CHT are subtle, concerted and synergistic according to TCM theory, which, to some extent, makes it difficult to assess its effects using traditional methods. Our results showed that CHT caused diverse biological changes in the urinary metabolome [25]. Many metabolic pathways were affected including those involving energy metabolism and amino acid metabolism and so on. The affected metabolites indicated that the overall metabolic response was the result of the complex effects of the CHT.

To overcome confounding inter-subject biochemical variation, 
Citation: You R, Yin L, Guan Y, Li L (2016) Rats' Metabolic Responses to Chronic Intervention with Cantonese Herbal Tea. Metabolomics 6: 178. doi:10.4172/2153-0769.1000178

Page 4 of 6

\begin{tabular}{|c|c|c|c|c|c|c|}
\hline \multirow{2}{*}{$\mathrm{m} / \mathrm{z}-\mathrm{RT}$} & \multirow{2}{*}{ Compound } & \multicolumn{4}{|c|}{ Change direction /VIP } & \multirow{2}{*}{ Metabolic pathway } \\
\hline & & Day 8 & Day 14 & Day 20 & Day 38 & \\
\hline M147T670 & Succinic acid & $\uparrow 6.5281$ & $\downarrow 2.0518$ & $\uparrow 3.9467$ & NS & \multirow{2}{*}{ Kreb's cycle } \\
\hline M273T1459 & Citric acid & NS & NS & $\uparrow 2.3470$ & $\uparrow 2.3734$ & \\
\hline M147T373 & Lactate & $\downarrow 3.0713$ & $\downarrow 1.7901$ & $\uparrow 1.4761$ & $\downarrow 1.8125$ & Glycosis \\
\hline M116T421 & Alanine & $\uparrow 4.5445^{\star}$ & $\downarrow 3.7234$ & $\downarrow 2.1962$ & $\downarrow 4.2176$ & Alanine metabolism \\
\hline M179T584 & Benzoic acid & $\downarrow 3.2421$ & $\downarrow 2.3833$ & $\uparrow 1.1397$ & $\downarrow 2.2444$ & Liver metabolism \\
\hline M147T458 & Oxalate & $\downarrow 1.2674$ & $\uparrow 1.9461$ & $\uparrow 2.7995$ & $\uparrow 3.0624$ & Others \\
\hline M147T389 & Glycolic acid & $\downarrow 1.6577^{*}$ & $\downarrow 1.9000$ & NS & $\uparrow 1.3567$ & Lipid oxidation \\
\hline M156T725 & Pipecolic acid & $\uparrow 1.4761^{*}$ & NS & NS & NS & Lysine degradation \\
\hline M218T608 & Methylglycine & $\uparrow 1.3604$ & $\downarrow 1.2153$ & NS & NS & Glycine and serine metabolism \\
\hline M241T699 & Uracil & $\uparrow 1.3095$ & $\downarrow 1.8046$ & NS & $\downarrow 1.8934$ & Pyrimidine metabolism \\
\hline M174T969 & N-Acetylglutamate & $\uparrow 1.1375^{*}$ & NS & NS & NS & N-Acetylglutamate \\
\hline M188T612 & Hypotaurine & $\uparrow 1.0497^{*}$ & NS & NS & $\downarrow 1.7514^{*}$ & $\begin{array}{c}\text { Taurine and } \\
\text { hypotaurine metabolism }\end{array}$ \\
\hline M142T650 & Proline & $\uparrow 1.0559$ & NS & NS & NS & Proline metabolism \\
\hline M174T616 & Ethanolamine & NS & $\downarrow 1.2123$ & $\downarrow 1.0327$ & NS & Ethanolamine metabolism \\
\hline M314T633 & Phosphate & NS & $\uparrow 9.1169$ & $\downarrow 9.5151$ & $\uparrow 1.3865$ & Phosphate metabolism \\
\hline M147T1056 & 2-hydroxyglutarate & NS & $\uparrow 2.2800$ & NS & $\uparrow 2.3190$ & Tryphanol metabolism \\
\hline M174T663 & Glycine & NS & NS & NS & $\uparrow 3.3763$ & Glysine and serine metabolism \\
\hline M218T882 & Aminomalonic acid & NS & NS & NS & $\uparrow 1.5326$ & The conversion of cysteine \\
\hline M292T697 & Glyceric acid & NS & NS & NS & $\downarrow 1.3571^{*}$ & Lipid metabolism \\
\hline M202T1632 & Indole-3-acetic acid & NS & NS & NS & $\uparrow 1.2082^{*}$ & Amino acid metabolism \\
\hline M313T1742 & Hexadecanoic acid & NS & NS & NS & $\uparrow 1.0891^{*}$ & Fatty acid biosynthesis \\
\hline M217T959 & Erythritol & NS & NS & $\uparrow 1.3895^{\star}$ & $\downarrow 1.2099$ & \multirow{2}{*}{ Others } \\
\hline M319T1579 & Galactose & NS & NS & NS & $\uparrow 1.1785^{*}$ & \\
\hline
\end{tabular}

"^"or " $\downarrow$ " indicates whether there was an increase or a decrease in metabolite concentrations compared to the control, respectively. "*” indicates a $p<0.05$, which is considered statistically significant. NS indicates that there was no significant change

Table 2: The metabolites that distinguish between the treated and control groups and their metabolic pathways.VIP indicates the relative contribution of the metabolite to the separation between groups.

repeated doses of CHT over a period of 38 days were administered. OPLS-DA models were constructed to identify time-dependent changes in metabolites related to $\mathrm{CHT}$ consumption by the comparison of treated rats to control rats at all sampling time points. The OPLS-DA models for comparing the CHT group with the control group at days 8 , 14 and 20 were robust and predictable, which indicated that prolonged ingestion of $\mathrm{CHT}$ resulted in a biological response and produced metabolic changes in the performance of the system. However, on day 26 the metabolic variation between the control and the dosed groups was reduced, compared to the models for days 8,14 , and 20, as indicated by the fact that the model for day 26 lacked predictability after permutation testing. This reduction was further indicated by the poor predictability of the OPLS-DA model for days 33, which had a negative $\mathrm{Q}^{2}$. The poor quality of these two models was perhaps suggestive of the attempts by the treated animals to regain homeostatic balance or to adapt to prolonged CHT ingestion [26]. It appeared that the rats' response to $\mathrm{CHT}$ intervention followed a response and recovery pattern [27]. However, the OPLS-DA model for day 38 could again produce an obvious differentiation between the groups, as indicated by the good $\mathrm{Q}^{2}$ and permutation testing, which suggested repeated ingestion of CHT produced gradual and cumulative changes in rats.

In support of these findings, the loading plots of the models for days $8,14,20$ and 38 showed that many different metabolites were associated with CHT intervention. More specifically, the endogenous metabolites responsible for differentiation fluctuated at different sampling time points. Due to this inconsistency, student's $t$-test was further used to choose the most significant metabolites. Integrating VIP statistics with $p$-values identified metabolites that could be considered the most important molecular markers separating the two experimental groups at different sampling time points. The metabolites most strongly influencing the differentiation between the control and treatment group urine samples also varied with time, which appeared to be an attempt to maintain homeostasis $[28,29]$ in response to mild stimuli. Compared to the metabolic profiles obtained from the controls on day 8, CHT intervention resulted in lower levels of glycolic acid and elevated levels alanine, hypotaurine, $\mathrm{N}$-acetylgultamate, pipecolic acid. Hypotaurine is a product of the enzyme cysteamine dioxygenase in the taurine and hypotaurine metabolic pathway and is an excellent 
Citation: You R, Yin L, Guan Y, Li L (2016) Rats' Metabolic Responses to Chronic Intervention with Cantonese Herbal Tea. Metabolomics 6: 178. doi:10.4172/2153-0769.1000178

scavenger. It may function as an antioxidant and a protective agent under physiological conditions [30]. The increase in hypotaurine levels may be associated with the antioxidative capacity of CHT stemming from its phytochemicals [31,32]. This is consistent with the observed elevation of hypotaurine after intake of green tea [33]. Glycolic acid may be produced from $\beta$-oxidation of fatty acids, which often leads to the generation of hydrogen peroxides, a cause of free oxidative damage. Decreased levels of glycolic acid, in concert with the elevation of hypotaurine, suggested that CHT intake is involved in the depletion of oxidation stresses. Pipecolic acid is the product of lysine degradation and is known to act as a neuromodulator in the central nervous system. Its increased concentration in urine samples suggested that the central nervous system may be modulated by the intake of CHT. N-Acetylglutamate (NAG) is biosynthesized from glutamic acid and acetyl-CoA by the enzyme NAGS. An elevation in alanine and $\mathrm{N}$-acetylglutamate levels might indicate that $\mathrm{CHT}$ intervention would result in the degradation of proteins to amino acids to regulate biological functions [34].

The same procedures were applied to the models at later time points on day 14 and day 20; however, the model characteristics were reduced, and the largest influence on the separation between the controls and the dosed rats was only an increase in the urinary excretion of erythritol on day 20 , with no other specific metabolic changes.

However, 38 successive days of CHT intake did lead to the downregulation of glyceric acid and hypotaurine and the up-regulation of indole-3-acetic acid, galactose and hexacanoic acid. Indole-3-acetic acid is produced from tryptophan by the action of certain bacteria, including Bacteriodes, Clostridia and E. coli, which catalyze tryptophan to tryptamine and indole-pyruvic acid, which is then converted to indole-3-acetic acid Hence, the increased urinary excretion of indolic compounds may reflect a variation in gut microbiota composition in response to the $\mathrm{CHT}$ intervention. It has been reported that drinking $\mathrm{Pu}$-erh tea may contribute to a microbial imbalance in the gut by reducing the number of beneficial bacteria [35]. It is possible that the elevation of galactose and hexacanoic acid may be associated with an imbalance of the gut microbiota because both can be absorbed by gut flora. Glyceric acid can be biologically derived from the oxidation of glycerol and some important intermediates of lipid metabolism, such as 2-phosphoglyceric acid and 3-phosphoglyceric acid [36]. The decreased concentration of glyceric acid perhaps indicated that CHT contributed to a decrease in lipid metabolism, which could be extrapolated to suggest that chronic ingestion of CHT caused a lower flow of energy metabolism. These negative metabolic effects were extended as a result of daily administration of herbal tea.

Based on the above observations, the modes of action of CHT are summarized in Figure 2. As illustrated, energy metabolism and amino acid metabolism are involved in the entire process; however, the whole process can be divided into two stages. The biological responses of the initial stage are characterized by an elevation in antioxidant activity and depletion of oxidative stress until days 8 , but this kind of influence diminishes over time, and CHT produced no obvious changes until days 33 . The biological responses in the second stage were typified by a decreased flow of energy metabolism and a gut microbiota imbalance, which indicated that repeated ingestion of $\mathrm{CHT}$ produced slight and cumulative effects on the urinary metabolome.

\section{Conclusion}

Through both the multivariate and univariate analyses, we show that the metabolic response to $\mathrm{CHT}$ is a dynamic process with

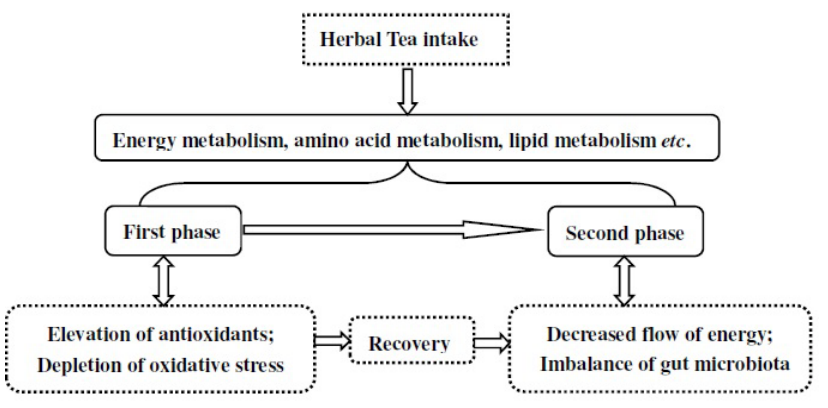

Figure 2: A summary of the modulating effects of $\mathrm{CHT}$ on rats over time.

multiple targets, as suggested by the observations based on different model characteristics and the identification of a complex series of discriminatory metabolites at different sampling time points during the course of the experiment. Fluctuation in metabolite levels over time may be the result of adaptations after prolonged ingestion. These changes provide potential target pathways from which an in-depth understanding of the action of the mechanism of $\mathrm{CHT}$ may be obtained.

\section{Acknowledgements}

This work is support by the National Natural Science Fund of China (20436020). We thank the Shenzhen Research Institute of the Hong Kong University of Science \& Technology for providing animal care facilities.

\section{References}

1. You R, Xu Z, Hu S, Li L (2012) Characterization of temporary metabolic changes following Cantonese Herbal Tea intervention. Phytother Res 26: 1097-1102.

2. You R, Pang Q, Li L (2014) A Metabolic Phenotyping Approach to Characterize the Effects of Cantonese Herbal Tea on Restraint Stressed Rats. Biol Pharm Bull 37: 1466-1474.

3. Deng J, Yang Y (2013) Chemical fingerprint analysis for quality assessment and control of Bansha herbal tea using paper spray mass spectrometry. Anal Chim Acta 785: 82-90.

4. Zhao J, Deng J, Chen Y, Li S (2013) Advanced phytochemical analysis of herbal tea in China. J Chromatogr A 1313: 2-23.

5. Clement YN, Morton-Gittens J, Basdeo L, Blades A, Francis MJ, et al. (2007) Perceived efficacy of herbal remedies by users accessing primary healthcare in Trinidad. BMC Complement Altern Med 7: 1-9.

6. Liu Y, Ahmed S, Long C (2013) Ethnobotanical survey of cooling herbal drinks from southern China. J Ethnobiol Ethnomed 9: 82.

7. Verpoorte R, Choi Y, Kim H (2005) Ethnopharmacology and systems biology: a perfect holistic match. J Ethnopharmacol 100: 53-56.

8. Nicholson JK, Lindon JC (2008) Systems biology: metabonomics. Nature 455 1054-1056.

9. Zhang J, Zhang Y, Du Y, Chen S, Tang H (2011) Dynamic metabonomic responses of tobacco (Nicotiana tabacum) plants to salt stress. J Proteome Res 10: 1904-1914.

10. Kinross J, Li JV, Muirhead LJ, Nicholson J (2014) Nutritional modulation of the metabonome: applications of metabolic phenotyping in translational nutritional research. Curr Opin Gastroenterol 30: 196-207.

11. Öhman A, Forsgren L (2015) NMR metabonomics of cerebrospinal fluid distinguishes between Parkinson's disease and controls. Neurosci Lett 594 36-39.

12. Wei T, Zhao L, Jia J, Xia H, Du Y, et al. (2015) Metabonomic analysis of potential biomarkers and drug targets involved in diabetic nephropathy mice. Sci Rep 5: 1-14.

13. Nicholson JK, Wilson ID (2003) Understanding'global'systems biology: metabonomics and the continuum of metabolism. Nat Rev Drug Discov 2: 668-676. 
Citation: You R, Yin L, Guan Y, Li L (2016) Rats' Metabolic Responses to Chronic Intervention with Cantonese Herbal Tea. Metabolomics 6: 178. doi:10.4172/2153-0769.1000178

14. Xie G, Zhao A, Zhao L, Chen T, Chen H, et al. (2012) Metabolic fate of tea polyphenols in humans. J Proteome Res 11: 3449-3457.

15. Gorlach S, Fichna J, Lewandowska U (2015) Polyphenols as mitochondriatargeted anticancer drugs. Cancer Lett 366: 141-149.

16. Ulaszewska MM, Trost K, Stanstrup J, Tuohy KM, Franceschi P, et al. (2016) Urinary metabolomic profiling to identify biomarkers of a flavonoid-rich and flavonoid-poor fruits and vegetables diet in adults: the FLAVURS trial. Metabolomics 12: 1-22

17. Gao X, Pujos-Guillot E, Sébédio JL (2010) Development of a quantitative metabolomic approach to study clinical human fecal water metabolome based on trimethylsilylation derivatization and GC/MS analysis. Anal Chem 82: $6447-$ 6456.

18. Vinaixa M, Schymanski EL, Neumann S, Navarro M, Salek RM, et al. (2015) Mass spectral databases for LC/MS and GC/MS-based metabolomics: state of the field and future prospects. Trac Trends in Analytical Chemistry 78: 23-35.

19. Lundstedt T, Hedenstr M, Soeria-Atmadja D, Hammerling U, Gabrielsson J, et al. (2010) Dynamic modeling of time series data in nutritional metabonomics-a powerful complement to randomized clinical trials in functional foods studies. Chemometr Intell Lab Syst 104: 112-120.

20. Beltrán-Debón R, Rodríguez-Gallego E, Fernández-Arroyo S, Senan-Campos O, Massucci FA (2015) The acute impact of polyphenols from Hibiscus sabdariffa in metabolic homeostasis: an approach combining metabolomics and gene-expression analyses. Food Funct 6: 2957-2966

21. Smith C, Want E, O'Maille G, Abagyan R, Siuzdak G (2006) XCMS: processing mass spectrometry data for metabolite profiling using nonlinear peak alignment, matching, and identification. Anal Chem 78: 779-787.

22. Weljie A, Newton J, Mercier P, Carlson E, Slupsky C (2006) Targeted profiling: quantitative analysis of $1 \mathrm{H}$ NMR metabolomics data. Anal Chem 78: 44304442.

23. Bjerrum J, Nielsen O, Hao F, Tang H, Nicholson JK, et al. (2010) Metabonomics in ulcerative colitis: diagnostics, biomarker identification, and insight into the pathophysiology. J Proteome Res 9: 954-962.

24. Wang Y, Holmes E, Nicholson JK, Cloarec O, Chollet J, et al. (2004) Metabonomic investigations in mice infected with Schistosoma mansoni: an approach for biomarker identification. PNAS 101: 12676-12681.
25. Koo LC (1989) Ethnonutrition in Hong Kong: Traditional dietary methods of treating and preventing disease. The Hong Kong Practitioner 11: 221-231.

26. Lan K, Jia W (2010) An integrated metabolomics and pharmacokinetics strategy for multi-component drugs evaluation. Curr Drug Metab 11: 105-114.

27. Wikoff WR, Kalisak E, Trauger S, Manchester M, Siuzdak G (2009) Response and recovery in the plasma metabolome tracks the acute LCMV-induced immune response. J Proteome Res 8: 3578-3587.

28. Nicholson J, Connelly J, Lindon J, Holmes E (2002) Metabonomics: a platform for studying drug toxicity and gene function. Nat Rev Drug Discov 1: 153-162.

29. Lindon JC, Holmes E, Bollard ME, Stanley EG, Nicholson JK (2004) Metabonomics technologies and their applications in physiological monitoring, drug safety assessment and disease diagnosis. Biomarkers 9: 1-31.

30. Fellman J, Roth E (1985) The biological oxidation of hypotaurine to taurine: hypotaurine as an antioxidant. Progress in Clinical and Biological Research 179: $71-82$.

31. Chan E, Lim Y, Chong K, Tan J, Wong S (2010) Antioxidant properties of tropical and temperate herbal teas. J Food Compost Anal 23: 185-189.

32. Nishimura T, Duereh M, Sugita Y, Yoshida Y, Higuchi K, et al. (2015) Protective effect of hypotaurine against oxidative stress-induced cytotoxicity in rat placental trophoblasts. Placenta 36: 693-698.

33. Law WS, Huang PY, Ong ES, Ong CN, Li SFY, et al. (2008) Metabonomics investigation of human urine after ingestion of green tea with gas chromatography/mass spectrometry, liquid chromatography/mass spectrometry and $1 \mathrm{H}$ NMR spectroscopy. Rapid Commun Mass Spectrom 22: 2436-2446.

34. Feng J, Liu H, Zhang L, Bhakoo K, Lu L (2010) An insight into the metabolic responses of ultra-small superparamagnetic particles of iron oxide using metabonomic analysis of biofluids. Nanotechnology 21: 395101.

35. Xie G, Ye M, Wang Y, Ni Y, Su M, et al. (2009) Characterization of pu-erh tea using chemical and metabolic profiling approaches. J Agric Food Chem 57 3046-3054.

36. Li HD, Zeng MM, Tan BB, Liang YZ, Xu QS, et al. (2010) Recipe for revealing informative metabolites based on model population analysis. Metabolomics 3 353-361. 